\title{
Stimulated emission of an atomic system under coherent excitation
}

\author{
A. G. Petrashen, N. V. Sytenko \\ ITMO University, 197101, St. Petersburg, Russia \\ apetrashen@mail.ru
}

PACS 32.60.+i, 32.10.Dk, 32.80.-t

DOI 10.17586/2220-8054-2015-6-4-562-569

The stimulated emission of a coherently excited atomic system was studied in the presence of a strong magnetic field.

Keywords: coherent excitation, density matrix, Liouville equation.

Received: 25 April 2015

Revised: 30 April 2015

\section{Introduction}

The possibility of collective spontaneous emission was first indicated by Dicke [1]. Later, these effects were detected in cesium atomic beams [2], as well as in ensembles of rubidium atoms $[3,4]$. The ideas of Dicke were subsequently developed in numerous publications (see for instance [5,6]), which included mostly bi-level systems with different ways of population inversion, possibly leading to superradiance - high intensity pulses, followed by a certain delay in relation to the inverse state formation time. It should be emphasized that generally speaking, population inversion is not a necessary condition for the creation of superradiance. This phenomenon can occur if a dipole moment is induced on the upper state [7,9]. This is known as induced superradiance. The present work is not directly related to superradiance as such. Below, within the framework of the ideas used for superradiance, we will investigate the collective polarization emission of a complex atomic system which has been placed in a strong magnetic field, the lower state of which, is populated by axially symmetric interactions.

\section{Statement of the problem}

In this paper, we consider the time dependance of the $3^{3} S_{1}$ state of the $H e$ atom, the excitation of which is performed by a $2^{3} P$ multiplet of this atom. It is assumed that the system has been placed in a magnetic field strong enough to break the thin bond of the P-multiplet under consideration. We will also assume that the $2^{3} P$ state is populated from the ground state of this atom by a proton or electron impact or other axially symmetric excitation sufficient to break the fine bond, and its symmetry axis makes an angle $\theta$ with the axis $O Z$ of the laboratory frame of reference. As a result of this excitation, the ordering of the angular moments of the $T_{0}^{0}$ population and the longitudinal alignment of $T_{0}^{2}$ will be induced on the $2^{3} P$-levels in the frame of reference associated with the anisotropy axis. Then, by introducing a parameter $\alpha=T_{0}^{2} / T_{0}^{0}$, which may be called the anisotropy parameter for the population efficiencies of the $2^{3} P_{j}$ state magnetic sublevels in the laboratory frame of reference $X Y Z$, one can write:

$$
\sigma_{j, m ; j^{\prime}, m^{\prime}}=\frac{1}{2 j+1} A^{(0)}\left(j, j^{\prime}, l, s\right)+\alpha \sum_{q}(-1)^{j^{\prime}+m^{\prime}}\left[\begin{array}{ccc}
j & j^{\prime} & 2 \\
m & -m^{\prime} & q
\end{array}\right] D_{0, q}^{(2)}(\theta) A^{(2)}\left(j, j^{\prime}, l, s\right)
$$


where $l$ and $s$ are the orbital and the spin moments, $D_{m, m^{\prime}}^{2}(\theta)$ elements of three-dimensional rotation [10], and $A_{j}^{(k)}$ depends on coefficients of vector moment addition:

$$
A^{(k)}\left(j, j^{\prime}, l, s\right)=(-1)^{j^{\prime}+s+k+l} \sqrt{(2 j+1)\left(2 j^{\prime}+1\right)}\left\{\begin{array}{lll}
l & l & k \\
j & j^{\prime} & s
\end{array}\right\} .
$$

The Hamiltonian operator of the atomic system placed in a strong magnetic field of strength $\mathcal{H}$ is defined by the orbital $\hat{L}$ angular momenta and $\hat{S}$ - the spin moments:

$$
\hat{H}=\hat{H}_{0}+\mu_{0} \mathcal{H}(\hat{L}+2 \hat{S})+\hat{V}
$$

where $\mu_{0}-$ is the Bohr's magneton, and operator $\hat{V}$ describes the excitation of $3^{3} S_{1}$ state, from the lower $2^{3} P$ ones (hereinafter referred to as the state of ' $b$ ' and ' $a$ ', respectively) by electromagnetic field polarization. This polarization makes an angle $\chi$ with the $O Z$ axis of the laboratory frame of reference as follows:

$$
\left(V_{a b}\right)_{m_{a}, m_{b}}=\left\langle j_{a} m_{a}\left|d_{z}\right| j_{b} m_{b}\right\rangle=\hbar \widetilde{\Omega}\left(W_{a b}\right)_{m_{a}, m_{b}},
$$

where $\widetilde{\Omega}(t)=E(t)\langle r\rangle / \hbar$ is the Rabi frequency, which depends on the value of electric field strength $\vec{E}(t)$ and $\langle r\rangle$ - reducible matrix element of operator $\hat{r}$. The elements of the $W_{a, b}$ matrix are defined by the regulations of the vector additions:

$$
\begin{aligned}
& \left(W_{a b}\right)_{m_{a}, m_{b}}= \\
& \hbar \Omega_{R}(-1)^{l_{a}+s+j_{b} l_{b}+1} \sqrt{\left(2 l_{b}+1\right)\left(2 j_{b}+1\right)}\left[\begin{array}{ccc}
j_{b} & 1 & j_{a} \\
m_{b} & 0 & m_{a}
\end{array}\right]\left\{\begin{array}{ccc}
l_{b} & l_{a} & 1 \\
j_{a} & j_{b} & s
\end{array}\right\}\left[\begin{array}{ccc}
l_{b} & 1 & l_{a} \\
0 & 0 & 0
\end{array}\right] .
\end{aligned}
$$

The excitatory electric field $E(t)$ leads to the polarization of the medium, which is characterized by a polarization vector $\vec{P}$. The value of this vector is proportional to the mean value of the operator of dipole moment $\hat{d}$, evaluated on the correlation density matrix:

$$
\vec{P}=-n_{0} \Im\left[\operatorname{Sp}\left(\rho_{a b} \widehat{d}\right)\right] .
$$

From the last relation, one can see that the polarization of the environment by the excitation process is defined by the correlation density matrix and hence, depends on its variation. In turn, the right hand side of the Liouville equation, which describes the variation of the density matrix, contains the Rabi frequency, which depends on the electric field strength. So, the mutual variation of the density matrix and the electric field is described by a system of self-consistent equations:

$$
\begin{gathered}
\frac{d}{d t} \widetilde{\rho}_{a a}(t)=\Gamma \widetilde{\rho}_{b b}(t)-\frac{i}{\hbar}\left(V_{a b}(t) \widetilde{\rho}_{b a}(t)-\widetilde{\rho}_{a b}(t) V_{b a}(t)\right), \\
\frac{d}{d t} \widetilde{\rho}_{b b}(t)=-\Gamma \widetilde{\rho}_{b b}(t)-\frac{i}{\hbar}\left(V_{b a}(t) \widetilde{\rho}_{a b}(t)-\widetilde{\rho}_{b a}(t) V_{a b}(t)\right), \\
\frac{d}{d t} \widetilde{\rho}_{a b}(t)=-\frac{\Gamma}{2} \widetilde{\rho}_{a b}(t)-\frac{i}{\hbar}\left(E_{a}-E_{b}\right) \widetilde{\rho}_{a b}(t)-\frac{i}{\hbar}\left(V_{a b}(t) \widetilde{\rho}_{b b}(t)-\widetilde{\rho}_{a a}(t) V_{a b}(t)\right), \\
\widetilde{\rho}_{b a}(t)=\widetilde{\rho}_{a b}^{\dagger}(t), \\
\nabla^{2} E-\frac{1}{c^{2}} \frac{\partial^{2}}{\partial t^{2}} E=\frac{4 \pi}{c^{2}} \frac{\partial^{2}}{\partial t^{2}} P
\end{gathered}
$$


where $E_{a}$ and $E_{b}$ are stationary energy levels of the lower and upper states and matrix $\Gamma$ describes the relaxation process. It should be mentioned that the $2^{3} P$ state of the helium atom consists of three levels, one of which with $j=2$ is metastable [11]. Since the lifetime of the upper $3^{3} S_{1}$ state is approximately one-third the size of the lower $2^{3} P_{j}$ state, this system of two multiplets can be considered self-contained, which means that the total population of levels ' $a$ ' ' $b$ ' is conserved.

In order to simplify the main calculation it is necessary to move the squared momentum and its projection from the base of the eigenfunction to the diagonalized base (M-base), which describes the integration with the strong magnetic field. Diagonalization was performed analytically using the Mathematica software package. As a result, two sets of orthogonal eigenvectors were obtained, each of which corresponds to the eigenvalues of the state ' $b$ ' $(\varepsilon)_{M_{b}}=\mu_{0} \mathcal{H} M_{b},\left(M_{b}= \pm 2.0\right)$ and the state ' $\mathrm{a}$ ' $(\varepsilon)_{M_{a}}=\mu_{0} \mathcal{H} M_{a},\left(M_{a}= \pm 3, \pm 2, \pm 1.0\right)$. In the latter case, the states with $M_{a}= \pm 1$ are doubly degenerate. Then, in light of the information above, the eigenvalues of the Hamiltonian operator in a strong magnetic field takes the form: $\left(E_{a}\right)_{M_{0}}=E_{a}+\left(\varepsilon_{a}\right)_{M_{0}},\left(E_{b}\right)_{M}=E_{b}+\left(\varepsilon_{b}\right)_{M}$.

In order to reduce the system (7) to a more convenient form for numerical computation, it is first necessary to introduce dimensionless time $\tau=t / \tau_{0}$, where $\tau_{0}=36 \cdot 10^{-9} \mathrm{sec}[11]$ - the lifetime of the excited $3^{3} S_{1}$ state, and secondly, to introduce the density matrix $\rho_{i, j}(t)(i, j=$ $a, b)$, connected with the analogous matrix used previously with the relations $\left(\rho_{i i}(\tau)\right)_{M, M_{1}}=$ $\left(\widetilde{\rho}_{i i}(\tau)\right)_{M, M_{1}}(i=a, b),\left(\rho_{b a}(\tau)\right)_{M, M_{1}}=\left(\widetilde{\rho}_{b a}(\tau)\right)_{M, M_{1}} \exp \left[-i \tau\left(\left(E_{b}\right)_{M}-\left(E_{a}\right)_{M_{1}}\right)\right], \quad\left(\rho_{i, j}(\tau)=\right.$ $\left.\left(\rho_{j, i}(\tau)\right)^{\dagger}\right)$. Additionally, it is assumed that the system is situated in a circular resonator, the eigenfrequency of which coincides with the transition frequency between the upper and lower multiplets. In order to eliminate rapidly oscillating terms in the system (7), the solution of the last equation of this system may be searched in the form of a progressive wave $E(t)=$ $-\mathcal{E}(t) e^{i \omega t}$, and the response of the system in the form of a superposition of such waves $P(t)=$ $\Re(\mathcal{P}(t)) e^{i \omega t}+i \Im(\mathcal{P}(t)) e^{i \omega t}$. Then, assuming that the field and polarization vector amplitudes are slowly varying, and that their values are independent of the space variables (mean field assumption), then system (7) takes the following form:

$$
\begin{gathered}
\frac{d}{d \tau} \rho_{a a}(\tau)=\Gamma_{a} \rho_{b b}(\tau)+\frac{i \Omega_{R}}{2}\left[\rho_{a b}(\tau)\left(W_{b a}\right)_{I}-\left(W_{a b}\right)_{I} \rho_{b a}(\tau)\right] \\
\frac{d}{d \tau} \rho_{b b}(\tau)=-\Gamma \rho_{b b}(\tau)-\frac{i \Omega_{R}}{2}\left[\left(W_{b a}\right)_{I} \rho_{a b}(\tau)+\frac{i}{2 \hbar} \rho_{b a}(\tau)\left(W_{a b}\right)_{I}\right] \\
\frac{d}{d \tau} \rho_{a b}(\tau)=-\frac{\Gamma}{2} \rho_{a b}(\tau)+\frac{i \Omega_{R}}{2}\left[\rho_{a a}(\tau)\left(W_{a b}\right)_{I}-\frac{i}{2 \hbar}\left(W_{a b}\right)_{I} \rho_{b b}(\tau)\right] \\
\frac{d}{d \tau} \rho_{b a}(\tau)=-\frac{\Gamma}{2} \rho_{b a}(\tau)-\frac{i \Omega_{R}}{2}\left[\left(W_{b a}\right)_{I} \rho_{a a}(\tau)+\frac{i}{2 \hbar} \rho_{b b}(\tau)\left(W_{b a}\right)_{I}\right] \\
\frac{\partial}{\partial t} \mathcal{E}=-4 \pi \omega n_{0} \Im \mathcal{P},
\end{gathered}
$$

where $\mathcal{P}=\operatorname{Sp}\left(\rho_{a b} r / a_{0}\right)$ and $n_{0}$ are the density of the particles. In order the last equation contains dimensionless values one can introduce the dimensionless Rabi frequency $\Omega_{R}=\tau_{0} \mathcal{E}\langle r\rangle / \hbar$. Than the last equation of the system (6) takes the form:

$$
\frac{d}{d \tau} \Omega_{R}=-\Omega_{M}^{2} \operatorname{Sp}\left(\rho_{a b} \frac{r}{a_{0}}\right)
$$


where the introduced denotation

$$
\Omega_{M}=\sqrt{4 \pi n_{0}\left(\frac{e a_{0}}{\hbar}\right)^{2} \hbar \omega \frac{\langle r\rangle}{a_{0}}},
$$

which depends on $\langle r\rangle$ - the dimensionless mean value of operator $\hat{r}$ in the state $3^{3} S_{1}$.

\section{The results of numerical calculation. Discussion}

Before considering the results of numerical calculations, it should be mentioned that the fine splitting for the $2^{3} P_{j}$ state is relatively small and that this splitting is due to the strong magnetic field, compared to the energy difference of the 'centers of gravity' of $2^{3} P_{j}$ and $3^{3} S_{1}$ multiplets, the radiation of the system under consideration should be similar to that of the bi-level system considered in detail in [8]. The emissions of such a system are harmonic oscillations, known as nutations. After this, the system was integrated using initial conditions $\Omega_{R}(0)=0$ and the density matrix values that were obtained in the previous step. The process of integration was accomplished via the series expansion method that was described in detail in our previous articles $[12,13]$. The results of our calculations are represented on the Fig. 1 Fig. 5.

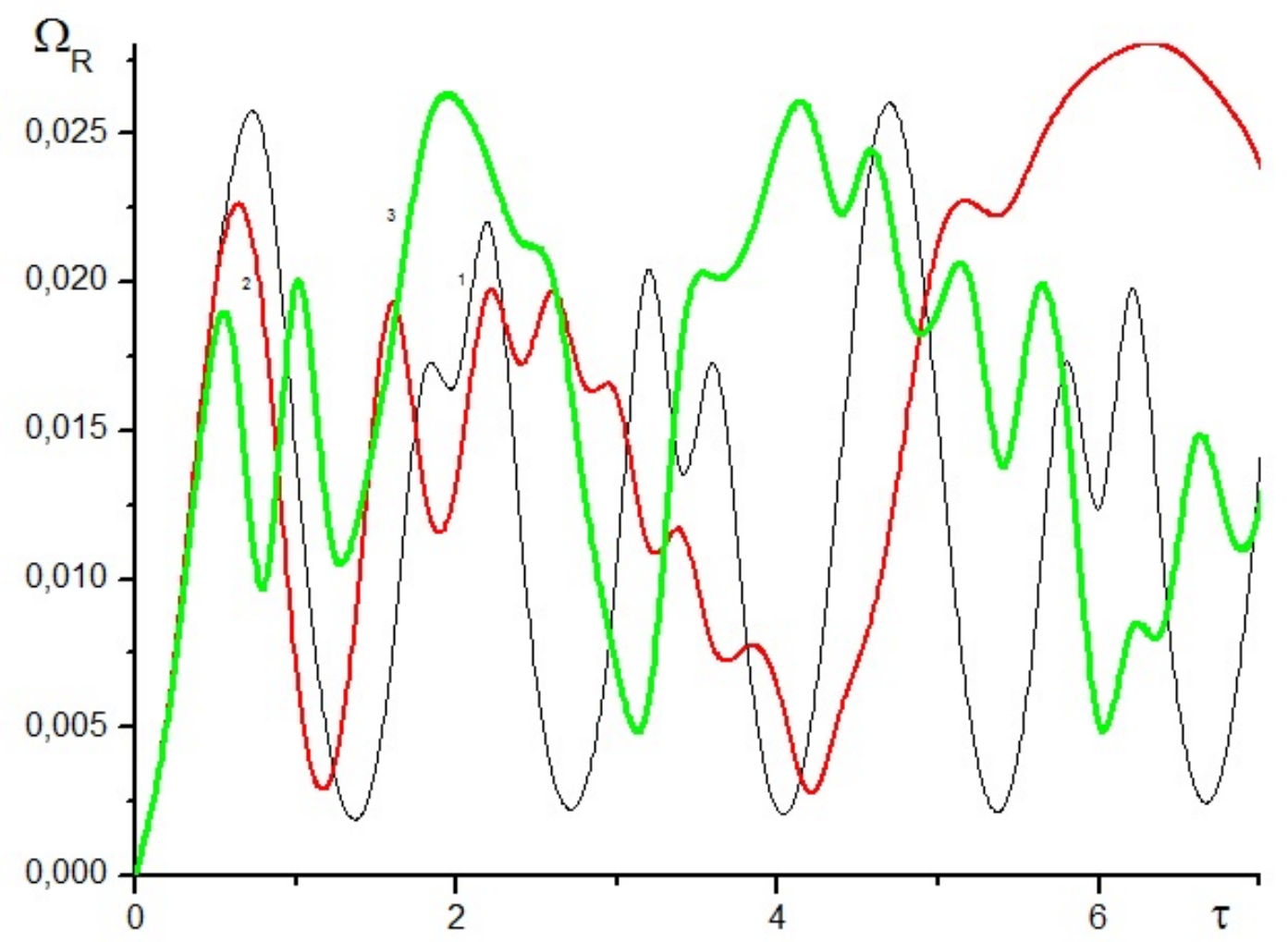

FIG. 1. The dependence of the induced Rabi frequency on dimensionless time for two values of particle density $n_{0}=10^{11}$ and $n_{0}=10^{13} \mathrm{~cm}^{-3}$

In Fig. 1, the dependence of the induced Rabi frequency on dimensionless time is shown for two values of frequency $\Omega_{M}$ (two values of particles density $n_{0}$ ). From this figure, one can see that the nutation frequency increases with increasing $\Omega_{M}$. Also, one can see that the nutations take place relative to some curve that shifts up the abscissa axis with increasing $\Omega_{M}$. 


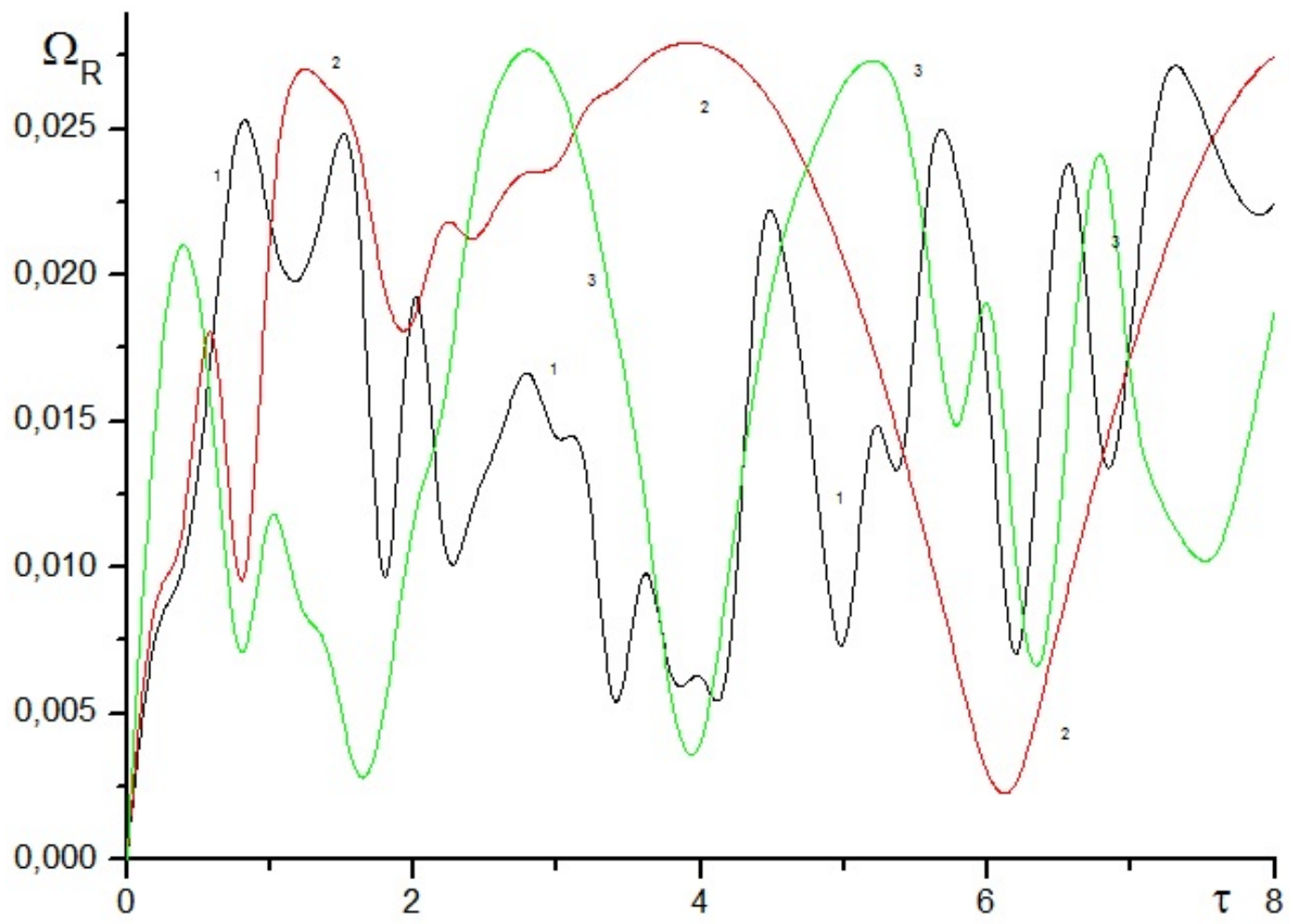

FIG. 2. The dependence of the induced Rabi frequency on dimensionless time for two laser detuning frequency values $\delta=0,0.5,1$

The dependence of the induced Rabi frequency upon time for some laser detuning frequency values is represented in Fig. 2. From this plot, one can see that at zero detuning, the dependence is represented by a sequence of rather short impulses. With increased signal cleaning, the widths of the impulses first rapidly increase, however, with additional increased detuning, the width of the impulses begins to decrease.

The dependence of induced Rabi frequency upon time for some values angle $\chi$ (the angle between the direction of the magnetic field and the $O Z$ axis of the laboratory frame of reference) is illustrated in Fig. 3. From this figure, one can see that over the range of angle $0<\chi<\pi / 3$ the emission signal increases and for $\chi>\pi / 3$ rapidly decreases, with the signal vanishing at $\chi=\pi / 2$. A similar dependence for systems under coherent excitation was reportedly fixed in our woks $[12,13]$. It should be mentioned that such a dependence follows from the fact that at $\chi=\pi / 2$, the system is in a state of coherent population trapping. In other words, the population at the state $3^{3} S_{1}$ is zero, and thus, the system did not absorb the electromagnetic radiation. The situation under consideration is illustrated on the Fig. 4, where the dependence of the $3^{3} S_{1}$ state's population for some values of angle $\chi$ is represented.

The problem is also illustrated here, in the frame of the approximate solution for system (9) in the framework of perturbation theory over the Rabi frequency. By restricting to first order perturbation theory over the Rabi frequency, one can write:

$$
\begin{gathered}
\frac{d}{d \tau} \varrho_{a b}(\tau)=\Omega_{R} \rho_{a a}(0)\left(W_{a b}\right)_{I}(\tau), \\
\frac{d}{d \tau} \Omega_{R}(\tau)=-\Omega_{m}^{2} \Im\left[\operatorname{Sp}\left(\rho_{a b} \frac{r_{b a}}{a_{0}}\right)\right] .
\end{gathered}
$$




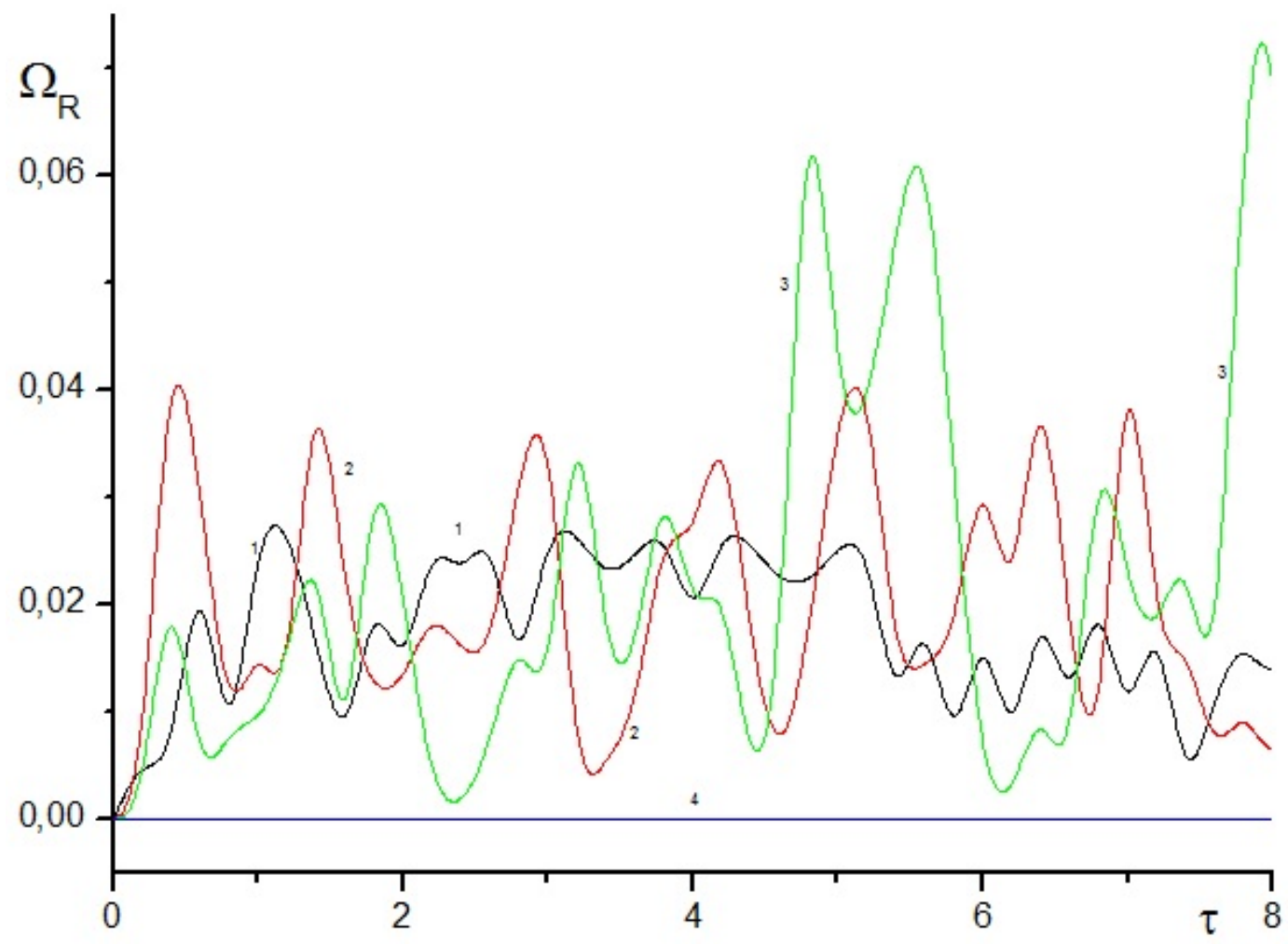

FIG. 3. The dependence of the inducted Rabi frequency on dimensionless time for two angle $\chi=0, \pi / 6, \pi / 3, \pi / 2$. Curves $1-4$

From these relations, it follows that:

$$
\frac{d^{2}}{d \tau^{2}} \Omega_{R}(\tau)=-\Omega_{R}\left\{\Omega_{m}^{2} \Im\left[\operatorname{Sp}\left(\rho_{a a}(0)\left(W_{a b}\right)_{I}(\tau) \frac{r_{b a}}{a_{0}}\right)\right]\right\} .
$$

The right hand part of this relation can be calculated with help of Mathematica. The resulting rather enormous relation takes a rather compact form in some cases. For instance, in the case when $\chi=\pi / 4$ or $\chi=\pi / 2$, one can write:

$$
\begin{gathered}
\frac{d^{2}}{d \tau^{2}} \Omega_{R}(\tau)+\frac{1}{144} \Omega_{M}^{2}\left(11+8 \cos \left[\frac{\Omega_{L}}{\hbar} \tau\right]\right) \Omega_{R}(\tau)=0, \quad \chi=\pi / 4 \\
\frac{d^{2}}{d \tau^{2}} \Omega_{R}(\tau)+\frac{7}{72} \Omega_{M}{ }^{2} \cos \left[\frac{\Omega_{L}}{\hbar} \tau\right] \Omega_{R}(\tau)=0, \quad \chi=\pi / 2 .
\end{gathered}
$$

Because of the assumption that only the induced emission was considered before, we are therefore suggesting that in the system at $\tau<0$, the dipole moment was induced. So, equations should be solved for the initial conditions $\Omega_{R}(0)=0.01, \frac{d}{d \tau} \Omega_{R}(0)=1$. The results are represented in Fig. 5, from which, one can see that induced emission at $\chi=\pi / 2$ is significantly lower than at $\chi=\pi / 4$. 


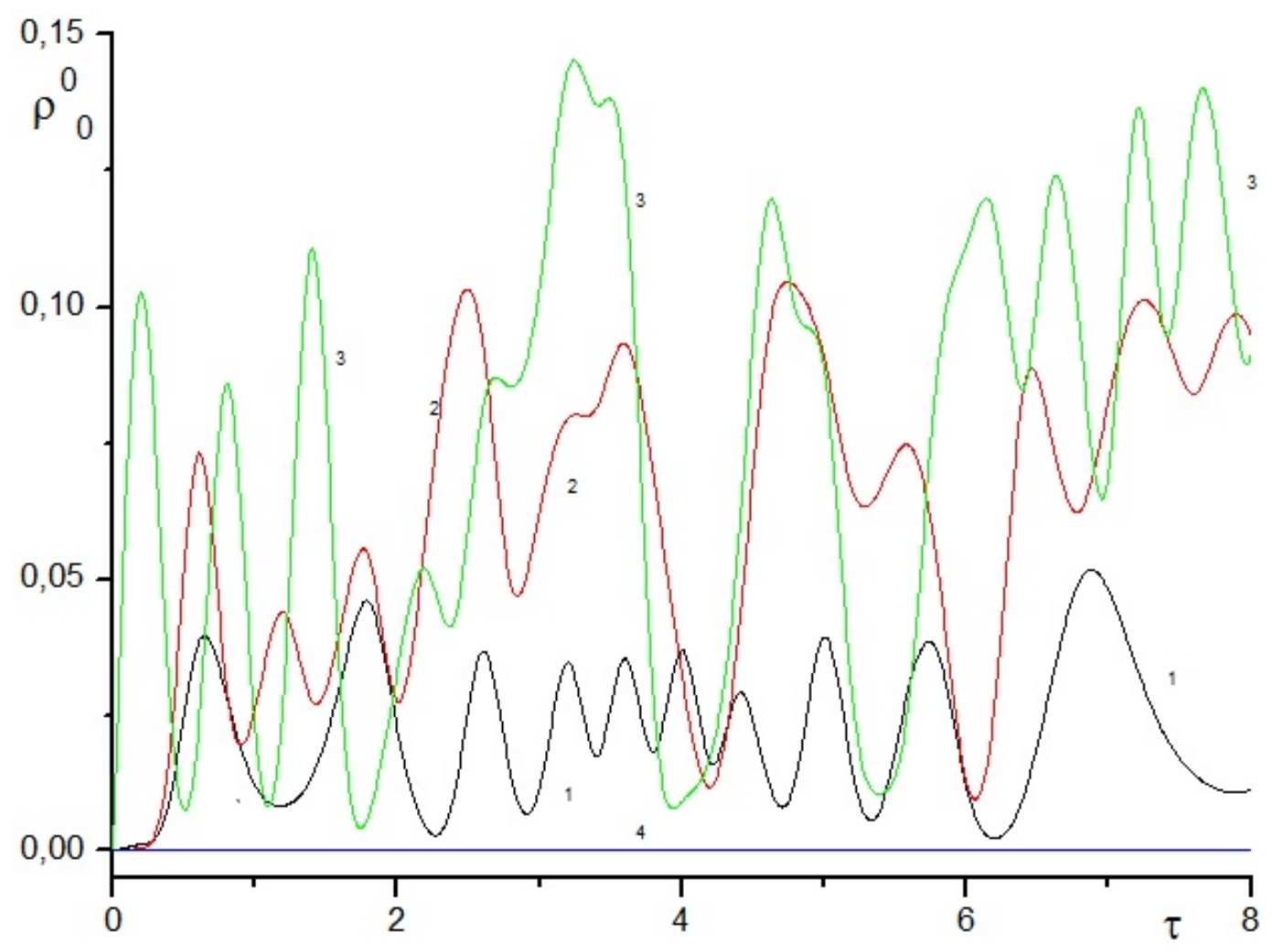

FIG. 4. The dependence of the $3^{3} S_{1}$ population for $\chi=0, \pi / 6, \pi / 3$ and $\pi / 2$. Curves $1-4$

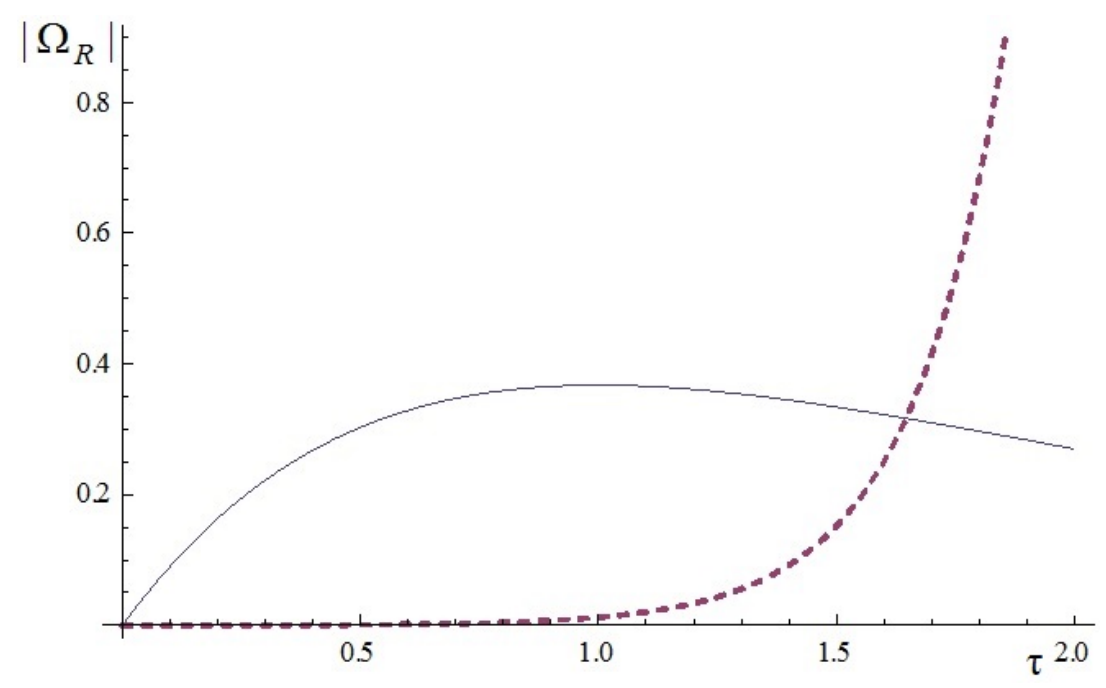

FIG. 5. The dependance on dimensionless time of of Rabi frequencies obtained in the frame of perturbation theory $\chi=\pi / 2$ (lower curve), $\chi=\pi / 4$ (upper curve) 


\section{Conclusion}

In this work, the induced emission of a complex atomic system in the presence of the strong magnetic field was considered. The dependence of the induced emission on the atom's density and laser's detuning was examined. It the case when the direction of the magnetic field is orthogonal to the direction of the polarization of exiting radiation the induced emission vanishes.

\section{Acknowledgements}

This work was partially financially supported by the Government of the Russian Federation (grant 074-U01), by Ministry of Science and Education of the Russian Federation (GOSZADANIE 2014/190, Projects No. 14.Z50.31.0031 and No. 1.754.2014/K), by grant MK5001.2015.1 of the President of the Russian Federation.

\section{References}

[1] R.H. Dicke Coherence in spontaneous radiation processes. Phys. Rev., 1954, 93, P. 99-105.

[2] H.M. Gibbs, Q.H. Vrehen, H.M. Hikspoors. Single-pulse superfluorescence in Cesium. Phys. Rev. Lett., 1977, 39, P. 547-550.

[3] E. Paradis, B. Barret et al. Observation of superfluorescent emissions from laser-cooled atoms. Phys. Rev. A, 2008, 77, P. 043419.

[4] G.O. Ariunbold, M.M. Kash, et al. Observation of picosecond superfluorescent pulses in rubidium atomic vapor pumped by 100-fs laser pulses. Phys. Rev. A, 2010, 82, P. 043421.

[5] R. Bonifacio, L.A. Lugiato. Cooperative radiation processes in two-level systems: Superfluorescence. II. Phys Rev. A, 1975, 12 (2), P. 587-592.

[6] J.H. Brownel, Y. Lu, S.R. Hartmann. Yoked superfluorescence. Phys. Rev. Lett., 1995, 75, P. $3265-3268$.

[7] R.F. Malikov, E.D. Trifatov. Induced superradiance in activated crystals. Opt. Comm., 1984, 52 (1), P. 74-76.

[8] L. Allen, J. Eberly. Optical resonancs and two-level atoms. New-York, London, 1975.

[9] A.I Zaiszev, V.A. Malyshev, I.V. Ryzhov, E.D. Trifonov. Inversion-free superfluorescence of the ensemble three-level in the high-Q rezonator. JETP, 1999, 115, P. 505-520 [In Russian].

[10] A. Jucys, A. Bandzaitis. Momentum theory in quantum mechanics. Vilnius, 1965, 462 p.

[11] A. Radzig, B.M. Smirnov. Reference data on atoms, molecules and ions. Springer-Verlag, Berlin, 1985.

[12] A.G. Petrashen, N.V. Sytenko. Polarization characteristics of emission of an ensemble of atoms on coherent excitation in the presence of a strong magnetic field with allowance for the Doppler profile width. Optics and Spectroscopy, 2015, 118 (6), P. 851-856.

[13] A.G. Petrashen, N.V. Sytenko. The polarization characteristncs of the radiation of atomic ensemble under the choherent exitation in the presence of the strong magnetic field. Nanosystems: Phys.,Chem., Math., 2015, 6 (1), P. 122-132. 